\title{
Tibetan Parents Self-cultural Identity Level for Middle School Students Trilingual Educational Impact Study
}

\author{
Linjia Gao ${ }^{1, a}$ \\ ${ }^{1}$ English Department of Sichuan Minzu College, Sichuan, Kangding, China \\ E-mail: glj@scun.edu.cn
}

Keywords: Self-cultural Identity Level; Trilingual Education; Parents of Middle School Students

\begin{abstract}
This survey conducts a research about the impact of parents' self-cultural identity level for middle school students' trilingual education in Garze Tibetan Autonomous Prefecture (GTAP) of Sichuan province. In this study we will be concentrating on effect of parent's ethnicity, cultural education on trilingual study and examine the correlation between them. We have used statistical regression analysis to establish correlation between the two and will conduct an ANOVA test to see if there is a difference in the impact due to various ethnic groups. The result of our study will provide valuable references for the future studies in this field.
\end{abstract}

\section{Introduction}

Language is the most important, unique, and almost universal human possession. It is remarkable in its complexity, species-specificity, versatility, ubiquity, and is referred to as an "extraordinary institution" (Gruyter, 2005). Language, more than anything else, is what makes us human. It appears that no communication system of equivalent power exists elsewhere in the animal kingdom. Any normal human child will learn a language based on rather sparse data in the surrounding world, while even the brightest chimpanzee, exposed to the same environment, will not.

Language is a symbol of nationality culture (Claire, 2000). Over the ages, richness of a language is one of the measures of a nation's/ civilization's richness. Culture is the soul and spirit of a nation; language is the carrier of culture. It is of great importance for humanity to inherit and develop its own cultures through language. As a country with 56 nationalities, China is no exception. Each nationality unfolds rich and colorful cultures of its own before our eyes. Therefore, trilingual education to the minority students becomes a must and necessary for the national minority students in order to maintain and develop their national cultures. Many children around the world grow up in multilingual environments. In fact, it has been speculated that the number of children exposed to more than one language from birth is similar, if not larger, than the number of those who speak only one language (Tucker, 1998). Although governments in many countries deliberately depict monolingualism as the norm, multilinguals are predominant not only in Asia and Africa but also in Europe and North America (Montanari, 2009).

There are several factors that could affect the student enrollment in trilingual education. In this paper, we have tried to assess the impact of parents' self-cultural identity level for middle school students' trilingual education in Garze Tibetan Autonomous Prefecture (GTAP) of Sichuan province. There are several factors that determine self-cultural identity of a person, like ethnicity, language, educational level, region and religious belief, etc. However, in our study, we will be specifically concentrating on effect of parent's ethnicity, educational level on trilingual study and examine the correlation between them. We have used statistical regression analysis to establish 
correlation between the two and will conduct an ANOVA test to see if there is a difference in the impact due to various ethnic groups. The result of our study will provide valuable references for the future studies in this field.

Brief introduction of Ganzi Tibetan Autonomous Prefecture. Garze Tibetan Autonomous Prefecture (GTAP) is located in western Sichuan of China with 153,000 square kilometers and 880,000 population, Tibetans account for $77.8 \%$ of the whole population. The capital city is Kangding. Generally speaking, three parts consist of the big tibetan region in China, there are respectively Tibet, Khamgpa and Amdo . Geographically, our prefecture is one of the major parts of Khampa region, which still includes other tibetan prefectures, like Muli of Liangshan、Changdu of Tibet、Yushu of Qinghai、 Diqing of Yunnan etc. But according to the local customs, the male of our prefecture is called Khampa hanzi and Sichuan Minzu College, which is located in in Garze Tibetan Autonomous Prefecture (GTAP) is called Khampa Daxue (university), where there exists a Khampa Culture Research Center in which the Tibetan scholars and professors are conducting various research subjects ranging from literature to math 、 from education to economy and so on. Therefore, we can see, to conduct the Tibetan-related topic like trilingual education in GTAP is of great importance in order to enrich Tibetan cultural study.

Parent's Self-cultural Identity. Cultural identification is the feeling of being included in a group with whom you associate yourself. This can be based on where you live, history, nationality, language, education and so on. Self-cultural identification may refer to people's sense of self culture and how the related to others (Xun Libo, 2012). There exist many factors to influence one's self-cultural identity such as the level of accepting education, the personal economic condition, and the place where he lives. This paper conducts a survey to prove that the higher level the parents accept education, the better economic condition they are and the more advanced place the live the stronger awareness the parents have to accept the trilingual education for their kids.

\section{Research Instruments}

Participants. The participants I chose are tibetan parents whose kids are studying in the middle school, 40 students are studying at the stage of senior school and 23,(30) students come from the junior at different grade. All of the participants come from different regions in GTAP prefecture (completely there are 18 counties in GTAP prefecture with various developed level).

Questionnaire Survey. For our study, we used a survey questionnaire to collect data. The sample for this survey was selected from parents of middle school students in GTAP.The design questionnaire is borrowed from previous researchers including Bob Adamson, Anwei Feng and Yi Yayuan) (Bob Adamson, Anwei Feng and Yi Yayuan, 2013)

The questionnaire contains three sections. The first section includes the personal information of the participants and his or her spouse. The second section conveys the knowledge that participants know about his or her child(ren)'s school, which we prepare 10 yes or no questions. The last part emphasizes on parents attitude towards language and language education in middle school.

Research Procedures. The research was conducted using statistical method. Secondary study on trilingual education in China and various other countries led us to narrow down the study to determine effect of parental cultural identity. The factors that determine cultural identity are - . Our hypothesis statement is that acceptance of trilingual education has positive correlation with a Tibetan parent's cultural identity. For the study we used a random sampling and survey techniques. The participants were selected from a stratified random sampling of seventy parents from different 
counties in GTAP. Survey questionnaire were sent to seventy participants and chief researcher worked with the participants to provide clarification. After the survey responses were received, we tabulated the data and analyzed it using regression analysis, using Minitab as the analysis tool.

\section{Results}

Effect of Parent's Educational Level on Attitude towards Trilingual Education. We had 70 numbers of parents in our sample data. The education level had following categories - primary school, junior middle school, senior middle school, technical school and university and higher education. Data had 17 parents in first category, 19 in second, 18 in third and one in fourth, and 16 in higher education category. Three parents didn't provide educational level and hence we had to reject those data from our study. The regression analysis provided the following equation:

$$
\text { Attitude }=1.08-0.0183 \text { parent education }
$$

This supported our hypothesis that there is a positive correlation between parent's education and open to embracing trilingual education for their kids. However, the correlation seems a weak correlation.

Effect of Parent's Ethnicity on Attitude towards Trilingual Education. The regression analysis provided the following equation:

$$
\text { Attitude }=4.55-0.225 \text { ethnicity }
$$

The Tibetans with a strong cultural identity have a strong affinity for trilingual education. However, the coefficient of correlation shows that, there is a weak or negative correlation with a correlation coefficient of 0.225

Owing to the two weak correlations between ethnicity and attitude, education level and attitude, we conducted interviews with Tibetan parents to supplement our study. Our interviews are mainly concerned with ethnicity.

Interview Questions. 1). Do you think you are Tibetan or Chinese? Why?

Mostly, they reply that they are Tibetan. The reasons can be concluded in the following points:

a. They were born in the Tibetan region, which is full of Tibetan atmospheres.

b. The different language.

c. The strong religious belief.

d. The living habit, eating habit and even entertainment habit etc.

2). What's your general view about your kids to learn Tibetan, Chinese and English in terms of their usefulness for their future?

To learn the three languages well is both important. Not only for finding a better job, but also for their personal development. So we can see, even the Tibetans have strong self cultural identification, still they want their kids become more competence in the future society and have a better future.

\section{Findings and conclusions}

1). No matter how low/high parents' education level is, they are willing to send their kids to trilingual study.

2). Even the people who have strong self-cultural identification are pleased to admit children to take part in the trilingual program. 
3). Parents actively support the trilingual education mostly for the sake of better future and better personal development for their kids.

\section{Limitation}

The weakness is that we concentrated it only on one category called Tibetans, we need to extend it to other categories and segment it further to see if any particular region has more influence. Having established, the strong affinity of Tibetans in general for trilingual education based on two variables we studied, we need to extend the effect other variables.

\section{References}

[1] Walter De Gruyter. (2005). Theoretical perspectives of trilingual education. Int's J. Soc Lang. 171, p.7.

[2] Claire Kramsch. (2000). Language and culture. Shanghai: Shanghai Foreign Language Education Press.

[3] Montanari, Simona. (2009). Pragmatic differentiation in early trilingual development. Int's J. Soc Lang. 36 p.597.

[4] Xianzhi. Fitch, W. Tecumseh. (2005). The evolution of language. Clevedon: Multilingual Matters.

[5]Http://baike.baidu.com/link?url=6LbHiy94dZO6RiaFg4y0uoz7lJkqsv-mmr88G5hr83fWiRGUV cJY7DDS-1Lu0_wgCQ79S3kGueh2qdXfbK5Ho6W9vx15a0yFkrgVRYQXLr9Sn-OTcWDM9Olxs pZT-MVc9I_38FT7p7yU7OOiiFmHiNJKgWCD-H9djV9sqAhf_Bi( accessed December 13, 2015)

[6] Xun Libo, Review and Discussion on the Research of the Cultural Identity and Identification of the Ethnic Minority, Heilongjiang Minzu Congkan,2012(5)

[7] Bob Adamson, Feng, Anwei(2013). Models of trilingual education in the People's Republic of China30-50. In Minority Languages and Multilingual Education: Bridging the Local and the Global. Dordrecht, New York and London: Springerp 